\title{
Atmospheric escape from the Earth during geomagnetic reversal
}

\author{
Olga O. Tsareva*,1 Eduard M. Dubinin² ${ }^{2}$ Helmi V. Malova ${ }^{3,1}$, Victor Yu. Popov ${ }^{4,5,1}$, \\ Lev M. Zelenyi ${ }^{1,6}$ \\ (1) Space Research Institute, Russian Academy of Sciences, 117997 Moscow, Russian Federation \\ (2) MPI für Sonnensystemforschung, Katlenburg-Lindau, Germany \\ (3) Lomonosov Moscow State University, Skobeltsyn Institute of Nuclear Physics, Leninskie gory 1, 119991 Moscow, \\ Russian Federation \\ (4) Lomonosov Moscow State University, Faculty of Physics, Leninskie gory 1, 119991, Moscow, Russian Federation \\ (5) National Research University “Higher School of Economics”, 101000 Moscow, Russian Federation \\ (6) Moscow Institute of Physics and Technology (State University), Institutskii per. 9, 141700 Dolgoprudnyi, Moscow \\ region Russian Federation
}

Article history: received August 26, 2019; accepted November 13, 2019

\begin{abstract}
We considered basic mechanisms of atmospheric particle acceleration and estimated the escape rates of ionospheric ions $\left(\mathrm{H}^{+}\right.$and $\left.\mathrm{O}^{+}\right)$during the geomagnetic field reversal. It is assumed that during the reversal the Earth's magnetic field deviates from the current dipole configuration, and the quadrupole component dominates. The standoff distance of the quadrupole magnetosphere is about of 3 Earth's radii and therefore a magnetic shielding protects the atmosphere from sputtering and ion pickup but not from the polar and auroral winds.
\end{abstract}

Keywords: atmospheric ion escape, polar wind, auroral wind, geomagnetic reversal, axisymmetric quadrupole.

\section{Introduction}

The geomagnetic field is not stable and undergoes long-term changes. Relatively long periods of evolutionary changes are followed by rapid geomagnetic reversals that last an average of the order of 250000 years [Glassmeier et al., 2009]. The last one, the Matuyama-Brunhes reversal, occurred 780000 years ago. The further geomagnetic reversal is expected geologically soon, in about 1600 years [Tsareva et al., 2018], which is currently confirmed by a weakening of Earth's magnetic field and a shifting of the magnetic poles. An open question for research is what happens to the atmosphere during the reversal process.

According to the widely accepted concept, the magnetosphere protects the planetary atmosphere from erosion by the solar wind. In the absence of induced and convective electric fields, ionospheric ions cannot be accelerated to energies above the escape energy necessary to overcome the gravitational pull of the planet. Atmospheres of unmagnetized Mars and Venus, on the contrary, are subject to erosion, since the interplanetary magnetic field (IMF) easily reaches their ionosphere. 


\section{Olga 0. Tsareva et al.}

Can we expect the loss of a significant part of the Earth's atmosphere during the polarity transition, when the magnetic field strength decays to about $10 \%$ or less of its present value? For example, Wei et al. [2014] have suggested that geomagnetic reversal could significantly enhance the oxygen escape rate and be responsible for the mass extinction. On the other hand, a number of researchers [Gunell et al., 2018; Strangeway et al., 2010; Barabash, 2010], contrary to the above paradigm, supposed that no, we cannot, since the escape rate of ions is determined by the total energy transferred from the solar wind into the ionosphere and is thus limited by the transfer efficiency and the size of the interaction region of the solar wind and magnetosphere [Barabash, 2010]. The present-day magnetosphere focuses the energy flux of the solar wind falling on the magnetopause into a small auroral zones. The energy flux in these zones is typically reaching values $10-100$ times higher than in the case with no magnetosphere. It increases the ionospheric outflow of heavy ions $\left(\mathrm{O}^{+}\right)$, but has little effect on the light ones $\left(\mathrm{H}^{+}\right)$ [Brain et al., 2013].

Gunell et al. [2018] have studied the dependence of the escape rate of ions on the magnetic moment of the planet. However, their rate estimates were obtained only for the magnetic moment of dipole field. According to the simulation [Glatzmaier and Roberts, 1995], during the reversal, the dipole component of the magnetic field decays with time and the quadrupole one can become dominant. In this paper we consider losses of the atmosphere of the Earth during the field reversals taking into account the quadrupole and the dipole components of the magnetic field. Since the quadrupole field also evolves in time, its magnitude and configuration at the reversal time may be random. And, for simplicity, we consider an axisymmetric quadrupole.

The main escape processes consist of Jeans escape, photochemical escape and sputtering for neutral particles [Brain et al., 2016], and ion pickup and polar wind for charged particles. The mechanisms - sputtering, photochemical or charge exchange escape [Tian et al., 2013] - described for neutral particle escape involve charged particles, therefore, a planetary magnetic field can influence their effectiveness.

Jeans escape is a classical thermal escape mechanism occurring when the energy of some particles in the high tail of distribution reach escape energy. Neutral atoms with energies exceeding the escape energy can also appear as a result of dissociative recombination of $\mathrm{O}_{2}^{+}, \mathrm{CO}^{+}$and $\mathrm{CO}_{2}^{+}$ions. Sputtering is a result of Coulomb collisions between exospheric neutrals and energetic ions. Fast-moving ions (of the solar wind or magnetosphere origin) can escape from a magnetic trap if they exchange charge with neutrals in the atmosphere (charge-exchange escape). Planetary ions which occur in plasma flow are picked up and can escape (pickup escape). Ions escaping through open magnetic field lines in the polar region and cusps correspond to polar and auroral winds, see sec. 3.1 and 3.2.

Jeans and photochemical escape processes can be excluded from the further consideration since they do not depend on the magnetic field strength and configuration. Photochemical escape is not significant for Earth, since the energy of O-fast atoms arising in process of the dissociative recombination on Earth is not sufficient to overcome its gravity.

There are two possible scenarios of atmospheric erosion during reversal, depending on the magnitude of the magnetic field. If the intrinsic magnetic field is weaker or equal to the induced one, then the planet's ionosphere is directly affected by the solar wind, similarly to Mars and Venus (unmagnetized planets). Ionized atoms of the exosphere (mainly $\mathrm{H}$ for Earth), accelerated by the convection electric field in the solar wind, escape by the pickup process. Pickup ions can also produce sputtering of neutrals. The induced magnetosphere also contains some analogs of 'polar caps' with expansion of the ionospheric plasma driven by the ambipolar electric field [Dubinin et al., 2017, 2011]

If the intrinsic magnetic field is stronger than the induced one, then it deflects the solar wind, thereby preventing ion pickup and sputtering, but contributes to the ions escape from the polar caps and cusps, i. e. the polar and auroral wind outflows. According to Slapak et al. [2017], only an insignificant part of the weakly accelerated $\mathrm{H}^{+}$ions can be captured by the magnetic field and returned to the atmosphere.

\section{Dayside magnetopause and plasmapause sizes}

\subsection{Magnetopause}

Changes in the magnetosphere and plasmasphere sizes due to long-term geomagnetic variations of the dipole component were considered by Glassmeier et al. [2004]; Siscoe and Chen [1975]. But since during the reversal it is 
assumed that the Earth's magnetic field deviates from the current dipole configuration, and the quadrupole component dominates, we estimated the magnetosphere and plasmasphere sizes for an axisymmetric quadrupole configuration.

The pressure balance between the dynamic pressure of the solar wind and the magnetic pressure of the magnetosphere (excluding atmospheric pressure) reads:

$$
p_{s w}=\left(B_{p}+B_{m c}\right)^{2} /\left(2 \mu_{0}\right)
$$

where $p_{s w}=\rho_{s w} v_{s w}^{2}$ denotes the solar wind pressure (Pa), $\mu_{0}=4 \pi \times 10^{-7} \mathrm{H} / \mathrm{m}$ is vacuum permeability $B_{p}$ is the planet's magnetic field ( $\mathrm{T}$ ) and $B_{m c}$ is the magnetic field of the magnetopause currents.

For a known magnetopause form, regardless of its scale, this sum can be represented as $B_{p}+B_{m c}=2 f_{0} B_{p}$, where $f_{0}$ is a form-factor. For the spherical magnetosphere a form-factor is $f_{0}=1.5$, and for a more realistic magnetopause shape (not a sphere) the coefficient $f_{0}=1.16$ [Voigt, 1995].

At the subsolar point of magnetopause, the dipole field strength is $B_{d i p}=\left|g_{1}^{0}\right|\left(R_{E} / R_{S, d i p}\right)^{3}$, and the quadrupole field strength is $B_{\text {quad }}=3 / 2\left|g_{2}^{0}\right|\left(R_{E} / R_{s, q u a d}\right)^{4}$, where $g_{1,2}^{0}$ are the leading dipole and quadrupole Gaussian coefficients taken for 2015 year $g_{1}^{0}=-29.4 \mu \mathrm{T}$ and $g_{2}^{0}=-2.5 \mu \mathrm{T}$ from the IGRF-12 model of Thebault et al. [2015], and $\mathrm{R}_{E}$ and $\mathrm{R}_{S}$ are the Earth's radius and the magnetopause standoff distance.

Solar wind pressure changes over time (see sec. 3.4), so the dipole magnetopause standoff distance varies in the range $R_{s, d i p}=6-15 R_{E}$. The quadrupole magnetopause standoff distance is:

$$
R_{S, \text { quad }}=\sqrt[4]{\frac{3}{2} \frac{g_{2}^{0}}{g_{1}^{0}}\left(\frac{R_{s, d i p}}{R_{E}}\right)^{3}} R_{E}=2.3-4.6 R_{E}
$$

The average magnetopause standoff distance of the dipole magnetosphere is $10 R_{E}$ at a solar wind speed of 500 $\mathrm{km} / \mathrm{s}$ and a density of 2.5 particles/ $\mathrm{cm}$. The average magnetopause standoff distance of the quadrupole magnetosphere under the same conditions is $3.36 R_{E}$. If the Earth magnetic field disappeared, the ionospheric plasma would stop the solar flux at a standoff distance of $R_{i m b}=1.2 R_{E}$ (i. e. where solar wind dynamic pressure balances the thermal pressure of the ionosphere $\left.p_{s w}=n k T\right)$. Since $R_{s, q u a d}>R_{i m b}$, the magnetic field protects the ionosphere from direct solar influence.

Figure 1 shows a dipole (a) and quadrupole (b) field lines, and combines models of a open and closed dayside magnetospheres. The field lines disturbed by the solar wind correspond to Voigt (1981) model of the dayside magnetosphere, whose magnetic field is $\mathbf{B}_{p}+\mathbf{B}_{c f}+\mathbf{B}_{i m f}$, where $\mathbf{B}_{p}$ is the intrinsic planet field (dipole and/or quadrupole), $\mathbf{B}_{c f}$ is the magnetic field of the Chapman-Ferraro current system and $\mathbf{B}_{i m f}$ - IMF. The quadrupole magnetospheres, their field lines topology and the system of large-scale currents were investigated by Vogt et al. (2004) using the MHD modeling.

\subsection{Magnetosphere and Plasmasphere}

In the open magnetosphere model, first proposed by Dungey (1961) for the southward IMF, the reconnection (or fusion) of interplanetary and geomagnetic field lines partially opens the Earth's magnetic field to the solar wind (Figure 1). The reconnection of the interplanetary and quadrupole field lines alternately opens the northern and southern lobes of the magnetic field when the IMF is directed southward and northward, respectively. Crosstail current flows along direction in northern lobe and against in southern one.

The solar wind electric field $\mathbf{E}_{s w}=-\left[\mathbf{v}_{S w} \times \boldsymbol{B}_{s w}\right]$ is related to the electric field of the magnetospheric convection $\mathbf{E}_{\text {convection }}=k \mathbf{E}_{s w}$ and can be derived from the scalar potential Stern (1973)

$$
\Phi_{\text {convection }}=k v_{s w}\left(B_{s w, z} y-B_{s w, y} z\right)
$$




\section{Olga 0. Tsareva et al.}

where $v_{s w}=500 \mathrm{~km} / \mathrm{s}$ is the solar wind speed assumed to be uniform, $B_{s w, z}=-5 \mathrm{nT}, B_{s w, y}=0 \mathrm{nT}$ are the components of IMF, $k$ is the efficiency of magnetic reconnection ( $k \sim 0.2$ [Levy et al., 1964]).

The magnetospheric convection potential is proportional to the size of the interaction region of the solar wind with the dayside magnetosphere, determined through its cross-sectional area $\pi h^{2}$, where $h$ is the maximum distance from the dayside magnetopause to the planet-sun line, shown in Figure 1. From the ratio of the cross-sectional areas of the dipole magnetosphere and half of quadrupole magnetosphere, taking into account that at any time only one lobe of the quadrupole magnetic field can be opened to the solar wind it follows that convection potential of quadrupole magnetosphere is $\Phi_{\text {convection }}^{\text {quad }}=h_{\text {quad }}^{2} / 2 h_{\text {dip }}^{2} \Phi_{\text {convection }}^{\text {dip }}=0.125 \Phi_{\text {convection }}^{\text {dip }}$. In a nonrotating Earth reference frame, the effective electrostatic potential includes the corotation potential $\Phi_{\Omega}$, which can be derived from the coefficients of the geomagnetic field spherical harmonics as follows:

$$
\Phi_{\Omega}=[\mathbf{W} \times \hat{\mathbf{r}}] \cdot \mathbf{A},[\mathbf{W} \times \mathbf{r}]=\Omega r \sin \theta \hat{\mathbf{j}},
$$

where $\mathbf{W}=2 \pi / 86400 \mathbf{z ~ s}^{-1}$ is the angular velocity of Earth's rotation, $\theta$ and $\phi$ are polar and azimuthal angles,
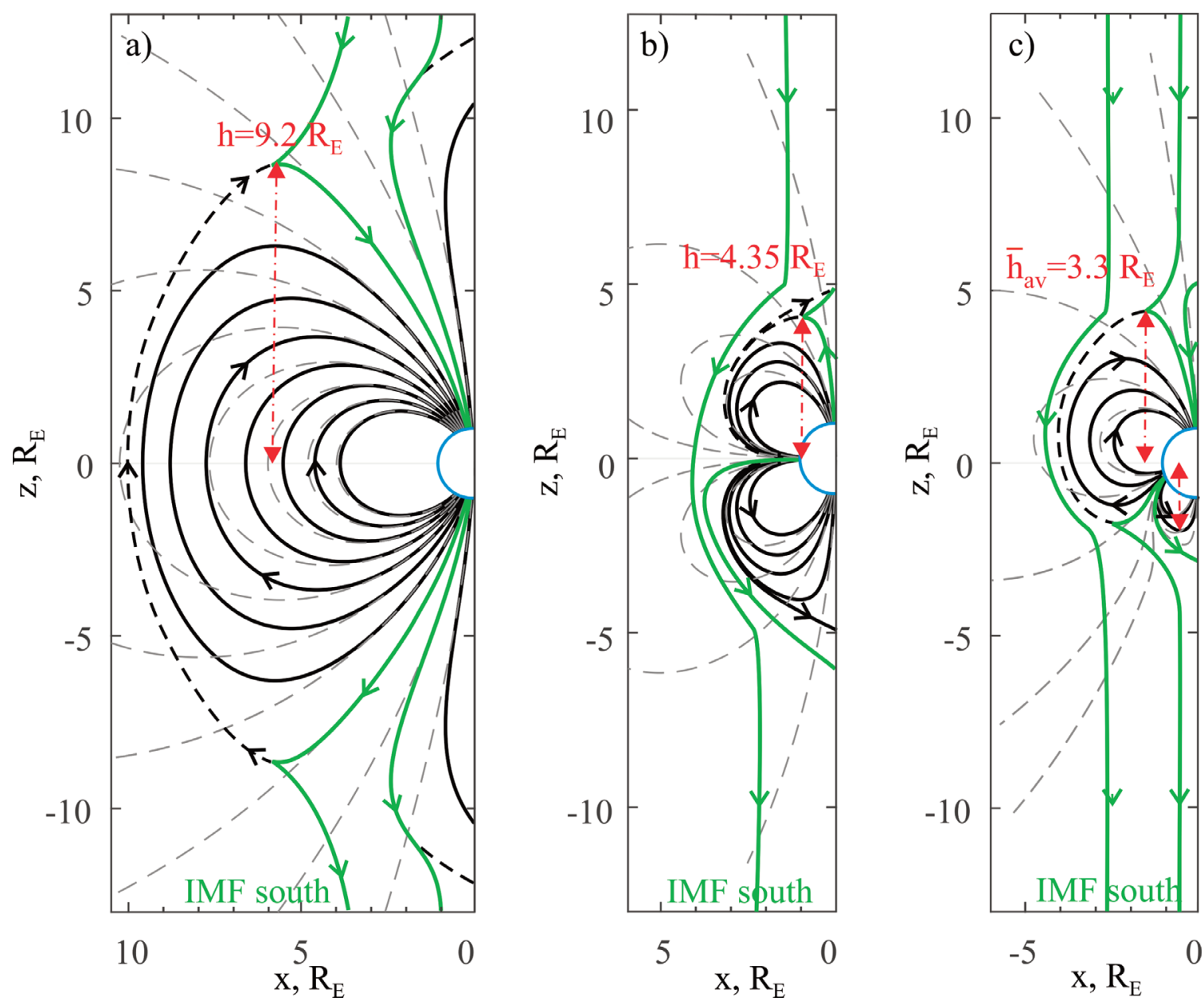

Figure 1. The field lines of the dipole (a), quadrupole (b) and mixed dipole-quadrupole (c) fields are perturbed (solid lines) and unperturbed (gray dashed) by the solar wind. Green lines are open field lines in the presence of the southward IMF. The height $h$ is the maximum distance from the dayside magnetopause to the planet-sun line, indicated by the red arrows in panels (a) and (b); $\bar{h}_{a v}$ is average of two heights indicated in panel (c). 
respectively. The vector potential $\mathbf{A}=\alpha \vec{\nabla} \beta$ of the dipole and quadrupole superposition is known from Tsareva [2019], where $\vec{\nabla} \beta=\theta \hat{\mathbf{j}} /(r \sin \theta)$ and

$$
\alpha(r, \theta)=R_{E}^{2} \sin ^{2} \theta\left[\left(\frac{R_{E}}{r}\right) g_{1}^{0}+\frac{3}{2}\left(\frac{R_{E}}{r}\right)^{2} g_{2}^{0} \cos \theta\right]
$$

hence $\Phi_{\Omega}(r, \theta)=\Omega \alpha(r, \theta)$.

The corotation electric field $\mathbf{E}_{\Omega}=-[\mathbf{W} \times \mathbf{r}] \times \mathbf{B}$ causes the cold particles to rotate eastward along with the Earth (at any field configuration) with velocity $\mathbf{v}_{E}=\left[\mathbf{E}_{\Omega} \times \mathbf{B}\right] / B^{2}$.

The effective electrostatic potential for the cold $\left(\mu=m v_{\perp}^{2} /(2 B)=0\right)$ particles motion is $\Phi_{\text {eff }}=\Phi_{\text {convection }}+\Phi_{\Omega}$. Remind that for hot particles of the plasmasheet, the potential of the gradient drift exceeds the corotational potential and leads to charge separation, i. e. the appearance of the electric field of polarization [Kivelson and Russell, 1995].

Figure 2 shows the equipotential lines $\Phi_{\text {eff }}$, along which charged particles drift. The last closed equipotential line of the convection electric field in the magnetosphere is a plasmapause. The plasmapause divides the azimuthal flow of dense cold plasma, which rotates around the Earth continuously and forms the plasmasphere, and the convective plasma flow directed from the magnetotail to the dayside magnetopause.

As can be seen from Figure 2, for the dipole configuration, the plasmapause field is located on the field lines at equatorial latitudes ( $\left.\Phi_{\Omega}^{d i p}\right|_{\theta=0}=-87 R_{E} / r \mathrm{kV}$ ). The nearest standoff distance to the plasmapause is $2.2 R_{E}$. There is one point in the dusk meridian, where the flow velocity is zero [Kivelson and Russell, 1995], which represents an exact balance between competing processes of convection and corotation. At this point, $\partial \Phi_{\text {eff }} / \partial r=0, \partial \Phi_{\text {eff }} / \partial \theta=0$ and $\partial \Phi_{\text {eff }} / \partial \varphi=0$, from where $r_{\text {zero-flow }}^{\text {dip }}=\sqrt{\Omega R_{E}\left|g_{1}^{0}\right| /\left(k_{\text {dip }} v_{s w}\left|B_{s w, z}\right|\right)}=5.2 R_{E}$.

For a quadrupole, there are two points on the dusk meridian. One point is located at the northern latitude of metastable circular orbit $\theta=63.4^{\circ}$ (Tsareva, 2019), when $B_{s w, z}<0$, and another one is located at the southern latitude $116.6^{\circ}$, when $B_{S W, Z}>0$. A standoff distance from the point to the quadrupole axis is $r_{\text {zero-flow }} \sin \theta=\sqrt[3]{3 / 2 \Omega R_{E}\left|g_{2}^{0}\right| 0.8 /\left(k_{\text {quad }} v_{s w}\left|B_{s w, z}\right|\right)} \sqrt{0.8}=2.5 R_{E}$. The nearest standoff distance of plasmapause from the axis is 1.3 .

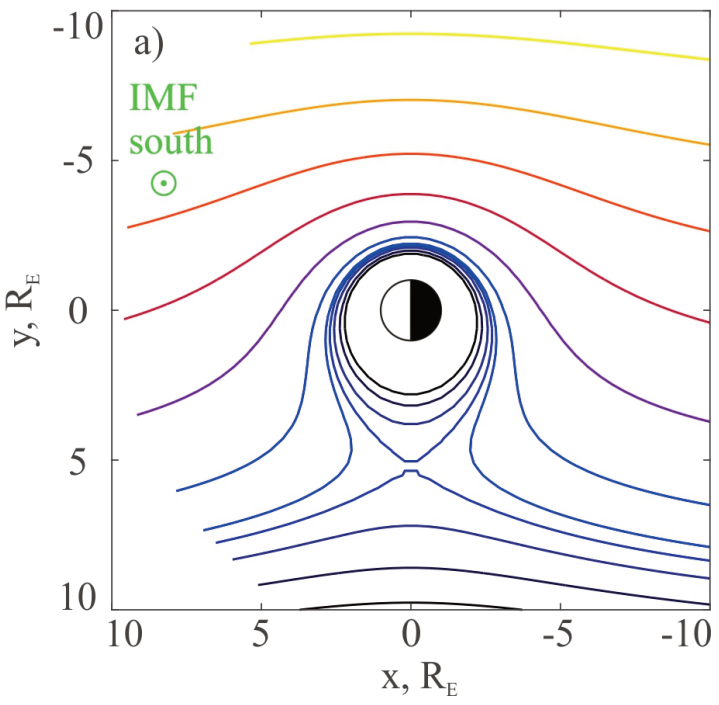

\section{Potential, $\mathrm{kV}$}

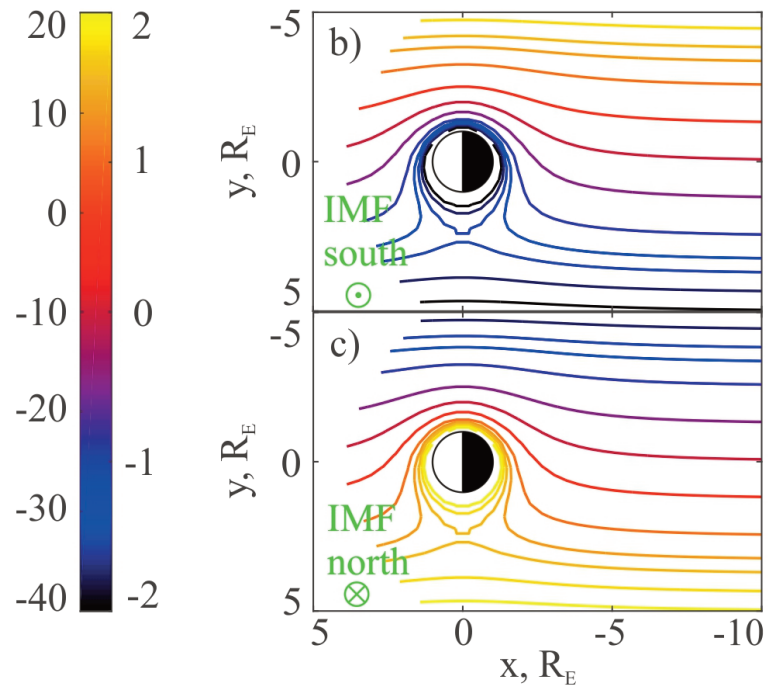

Figure 2. Equipotential contours of convection and corotation electric fields in dipole (a) and quadrupole (b) configurations at solar wind speed $v_{s w}=500 \mathrm{~km} / \mathrm{s}$. (a) in a plane $z=0$, IMF is directed southward, $B_{s w, z}=-5 \mathrm{nT}$; on a cone $z= \pm \sqrt{x^{2}}+y^{2} / \tan 63.4^{\circ}$, IMF is directed either southward (b) or northward (c). The reconnection of the interplanetary and quadrupole field lines alternately opens the northern and southern lobes of the magnetic field, respectively. 


\section{Olga 0. Tsareva et al.}

\subsection{Plasmaspheric plumes and wind}

The plasmasphere expands during quiet times and then, during a main phase of a magnetospheric storm, is eroded by convection, producing sunward extending plumes. During the storm's recovery phase, the plasmasphere's outer region of a low density is refilled by an upward ionospheric plasma. At high geomagnetic activity the enhanced magnetospheric convection removes $\sim 50 \%$ of mass of the plasmasphere. The mass fluxes in the plumes are $10^{25}-10^{27}$ ions/s [Andrè and Cully, 2012; Welling et al., 2015], much of which is lost via dayside reconnection. There is also a plasmaspheric wind that provides steady transport of cold plasma across the field lines from the plasmasphere with escape rate of $10^{25} \mathrm{ions} / \mathrm{s}$.

Since the entire quadrupole plasmasphere lies inside the magnetosphere, i. e. $r_{\text {zero-flow }}^{\text {quad }}<R_{s, \text { quad }}$, the plumes are formed within the magnetosphere with the same intensity and regularity as in present field configuration. Thus, these loss mechanisms are weakly dependent on the magnetic field [Gunell et al., 2018], but contribute to the total ion escape rate.

\section{Ion acceleration mechanisms}

The main sort of ions flowing from the ionosphere are singly charged oxygen $\left(\mathrm{O}^{+}\right)$, hydrogen $\left(\mathrm{H}^{+}\right)$and helium $\left(\mathrm{He}^{+}\right)$. Ions escaping from the polar caps can be accelerated to keV energies in the cusp, or can remain cold drifting in the magnetotail [Nilsson et al., 2012]. We divided ion outflows into two categories: "classical polar wind" and "auroral wind" driven by solar radiation and by localized solar wind energy dissipation, respectively.

Ion outflows vary with solar EUV flux (F10.7 index), solar wind pressure $\left(p_{s w}\right)$ and geomagnetic activity (Kp index). Higher X-ray and EUV radiation leads to an expansion of exosphere and higher escape rates at solar maximum or when the Sun was young [Lammer et al., 2018]. Solar wind dynamic pressure also plays a significant role in delivering energy to the ionosphere. Fok et al. [2005] found the gross dependence of auroral wind outflows $\left(\mathrm{O}^{+} / \mathrm{s}\right)$ on solar wind dynamic pressure $(\mathrm{nPa})$ fluctuations as $\log \left(Q_{a w, 0+}\right)=25.97 \pm .64+.61 \log \left(p_{s w}\right)$.

\subsection{Polar wind}

The source of acceleration for the polar wind ions is an ambipolar (polarization) electric field that forms when the faster and mobile electrons begin to separate from the ions. It is affected by solar thermal irradiation (illumination). The polar wind is considered in the context of global ionospheric convection, with Joule dissipation and the precipitation of soft electrons $(<500 \mathrm{eV})$ effects on the ambipolar electric field [Ogawa et al., 2000; Strangeway et al., 2005]. Ions in the polar wind are convected into the plasma sheet and then sunward, replacing the denser plasma being removed toward the subsolar magnetosphere.

In the polar caps, we observe polar wind ions with escape rate $Q_{0, p w, H}^{d i p}=7.8 \times 10^{25} \mathrm{H}^{+} / \mathrm{s}$ [Engwall et al., 2009] and $Q_{0, p w, O}^{d i p}=8 \times 10^{24} \mathrm{O}^{+} / \mathrm{s}$ [Yau and Andrè, 1997].

The escape rate of ions from the polar caps is proportional to their area [Gunell et al., 2018] and can be expressed through the ratio

$$
Q_{p w, \alpha}=Q_{0, p w, \alpha} \frac{S_{q u a d}}{S_{d i p}}=Q_{0, p w, \alpha} \frac{2 \pi r_{e x o, q}^{2} \Theta_{q u a d}}{2 \pi r^{2}{ }_{e x o, d} \Theta_{\text {dip }}},
$$

where $\alpha$ is the sort of ions, $r_{\text {exo }}=6871 \mathrm{~km}$ is the exobase radius, defined as the altitude where the atmospheric scale height is equivalent to the mean free path. The exobase distance can increase to $7371 \mathrm{~km}$ at high solar activity. Position of the exobase does not change with a change of the field topology $\left(r_{\text {exo,q }}=r_{\text {exo,d }}\right)$.

Assuming the average standoff distance to the dipole and quadrupole magnetopause, the ratio of the areas of open field line regions is $\Theta_{\text {quad }} / \Theta_{d i p}=0.112 / 0.11=1.01$ (see Appendix A). Thus, the total area of the polar caps and equatorial belt of the reversing quadrupole field almost coincides with the area of the polar caps of the present dipole field, therefore the escape rate of polar wind ions will change slightly. We note that in the quadrupole 
configuration, the area of the equatorial belt is 2 times higher the area of both polar caps.

If we consider the pure dipole magnetic field with the strength less in 10 times than the present value, then the average subsolar magnetopause standoff distance will be $R_{s, \text { rev.dip }}=\sqrt[3]{0.1} R_{s, d i p}=4.6 R_{E}$, and the area of the polar caps will increase in $\Theta_{\text {rev.dip }} / \Theta_{\text {dip }}=2.23$ times.

\subsection{Auroral wind}

The energization of ions in the cusp is caused by their resonant heating by broadband low-frequency electric field fluctuations (BBELF), which promote the formation of super-thermal and energetic upwelling ion population in the form of ion beams and conics [Yau and Andrè, 1997]. Upwelling is the most stable ion outflow from the cusp, and is dominated by $\mathrm{O}^{+}$ions. It displays the effects of both parallel and perpendicular energization to energies from one to tens of eV. Pollock et al. [1990] estimated the outflow rate of upwelling ions from two hemispheres $Q_{0, u p w, 0^{+}}$ $=2 \times 10^{25} \mathrm{ions} / \mathrm{s}, Q_{0, u p w, H^{+}}=0.5 \times 10^{25} \mathrm{ions} / \mathrm{s}$ and $Q_{0, u p w, H e^{+}}=0.1 \times 10^{25} \mathrm{ions} / \mathrm{s}$. We took these escape rates as average auroral wind rates.

It is not known whether BBELF waves are generated locally or propagate from the boundary of the magnetospheric region [Bouhram et al., 2004]. However, since transverse ion heating occurs in a wide range of heights and is partially controlled by the components of the IMF [Miyake et al., 2000], it is assumed that the waves responsible for heating the ions can be generated in the dayside reconnection region. The energy of these waves with a frequency equal to the ion gyrofrequency $\left(<10 \mathrm{~Hz}\right.$ for $\mathrm{O}^{+}$at altitude of $2.5-6.5 R_{E}, 0.2-4.0 \mathrm{~Hz}$ for $\mathrm{H}^{+}$at altitude of $6-9 R_{E}$ [Le et al., 2001], is transmitted by plasma instabilities, as well as by the Poynting descending flux to a lower altitude in the ionosphere cusp.

The escape rate of auroral wind outflows is proportional to the area of cusp, which is part of the polar cap, i. e. proportional its cross section $\pi h^{2}$ [Gunell et al., 2018], and the energy flux of the solar wind falling on the magnetopause. The escape rate increases with the magnetic moment of a dipole field until it reaches the maximum ion flux provided by the ionosphere. The maximum ion flux $\mathrm{O}^{+}$, measured by the $\mathrm{DE}-1$ spacecraft during the high solar and magnetic activity and integrated over both polar caps, is about $3 \times 10^{26}$ ions/s [Yau and Andrè, 1997]. This upper limit, reached by the outflow rate $\mathrm{O}^{+}$, makes it possible to judge the effectiveness of the magnetospheric shield.

The escape rate of auroral wind outflows for the quadrupole configuration can be estimated as

$$
Q_{\alpha w, \alpha}=Q_{0, u p w, \alpha} \frac{\pi h_{\text {quad }}^{2}}{\pi h_{\text {dip }}^{2}} \frac{S_{\text {quad }}}{S_{\text {dip }}} .
$$

The areas of open field line regions in the two field configurations are almost the same $S_{\text {quad }} / S_{\text {dip }}=1.01$. And the ratio of the cross sections of the magnetospheres is $h_{\text {quad }}^{2} / h_{\text {dip }}^{2}=0.22$ (Figure 1). Thus, the escape rate of auroral wind ions is reduced by 4.5 times.

For a dipole field with reduced strength of 10 times, this ratio is $h_{\text {rev.dip }}^{2} / h_{d i p}^{2}=0.21$, but with $S_{\text {rev.dip }} / S_{\text {dip }}=2.23$, the escape rate of auroral wind outflows will decrease by about 2 times.

\begin{tabular}{lcll} 
Ions Escape & Present-day Dipole & Reversing Quadrupole & Weak Dipole \\
\hline Auroral wind, H/s & $5 \times 10^{24}$ & $1.1 \times 10^{24}$ & $2.3 \times 10^{24}$ \\
\hline Polar wind, H/s & $7.8 \times 10^{25}$ & $7.9 \times 10^{25}$ & $1.7 \times 10^{26}$ \\
\hline Auroral wind, O/s & $2 \times 10^{25}$ & $4.4 \times 10^{24}$ & $9.4 \times 10^{24}$ \\
\hline Polar wind, O/s & $8 \times 10^{24}$ & $8.1 \times 10^{24}$ & $1.8 \times 10^{25}$ \\
\hline Total escape, $\mathbf{~ k g / s ~}$ & $\mathbf{0 . 9}$ & $\mathbf{0 . 5}$ & $\mathbf{1 . 1}$ \\
\hline
\end{tabular}

Table 1. Escape rates of ionospheric hydrogen and oxygen ions for the present dipole field, quadrupole reversing field and weak dipole (i.e. with magnetic field strength ratio $g_{1}{ }^{0} / g_{2}{ }^{0}=0.8$ ). 


\section{Olga 0. Tsareva et al.}

\subsection{Dipole-quadrupole Magnetosphere}

The field lines of the dipole-quadrupole magnetic field are defined by from Eq. (5). The latitudes of the polar caps boundaries $(\theta)$ satisfy the equality $\alpha\left(r_{\text {exo }}, \theta\right)=\alpha\left(R_{S}, \theta^{(\mp)}\right)$ where $\theta^{(\mp)}$ are the latitudes of metastable circular orbits [Tsareva, 2019]. The solution of this equation is shown in the Figure 3.

At $g_{1}^{0} / g_{2}^{0}=0$ the magnetic configuration is pure quadrupole one, there are three regions - a two polar caps and an equatorial belt, in which the field lines are open and connect to the solar wind. As the ratio $g_{1}^{0} / g_{2}^{0}$ increases, the equatorial belt moves to the southern polar cap where they connect at a point, marked by vertical line in the Figure 3 . After this line, the dipole component dominates in the magnetic field, the two polar caps, asymmetrical in size due to the quadrupole contribution, become smaller until they become equal.

Vogt et al. [2007] illustrated the change in the boundaries of the polar caps with an increase in the quadrupolar contribution to the dipole-quadrupole magnetosphere. They looked at several values of the dipole moment, the minimum of which one is about one-tenth of its present value. We have considered a change in the polar caps boundaries with a decrease in the dipole moment down to zero (Figure 3).

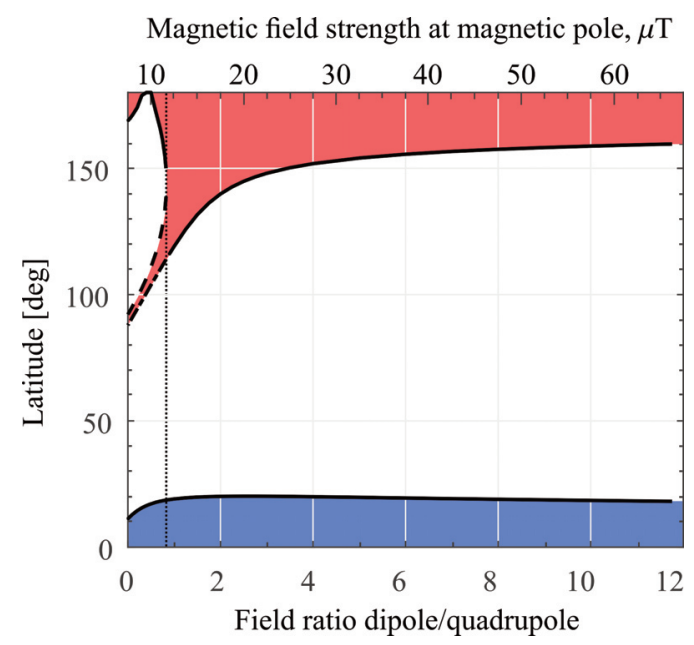

Figure 3. Polar cap boundary latitudes as a function of $g_{1}^{0} / g_{2}^{0}$ (dipole and quadrupole magnetic field strength ratio).

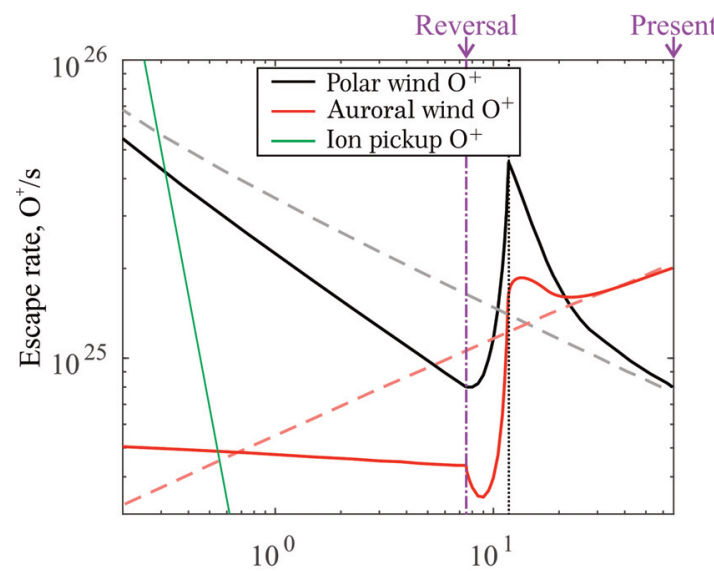

Magnetic field strength at magnetic pole, $\mu \mathrm{T}$

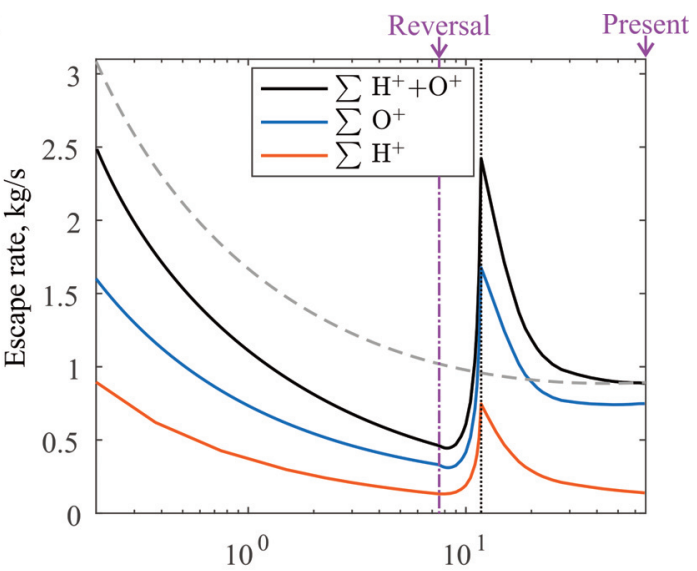

Magnetic field strength at magnetic pole, $\mu \mathrm{T}$

Figure 4. Hydrogen and oxygen ion escape rates as a function of magnetic field strength at the magnetic pole. Magnetic field is an axisymmetric superposition of dipole and quadrupole fields (solid lines) or a pure dipole field (dashed lines) as in the work of Gunell et al. (2018). Left: Oxygen ion escape rates for polar and auroral winds and ion pickup; Right: Total (polar and auroral) ion escape rates (without ion pickup). 
The ion escape rates from the polar caps are proportional to their sizes depending on the magnetic field. Figure 4 shows the $\mathrm{H}^{+}$and $\mathrm{O}^{+}$ion escape rates as a function of magnetic field strength at the magnetic pole. Solid and dashed curves correspond to the mixed dipole-quadrupole configuration and purely dipole configuration considered by Gunell et al. [2018]. Purple vertical line shows the reversing magnetic field without a dipole component. The total escape rate grows with decreasing magnetic field strength, but drops sharply when the field configuration is reversed and then increases again.

\subsection{Ion pickup}

Scenario when the solar wind is in a direct contact with the Earth atmosphere/ionosphere was considered by Wei et al. [2014] similar as was done for the unmagnetized Mars [Lundin and Dubinin, 1992]. The estimatel was based on the momentum transfer between the solar wind and planetary plasmas. The (accelerated) escaping magnetospheric flux (E) is related to the incident solar wind flux (sw) and the local (decelerated) solar wind flux (swE) as:

$$
Q_{\text {pickup }}=\delta \frac{v_{s w} m_{s w}}{v_{E} m_{E}}\left(n_{s w} v_{s w}-\frac{v_{s w_{E}}}{v_{s w}} n_{S w_{E}} v_{s w_{E}}\right) A=n_{E} v_{E} A
$$

where $v, m, n$ is speed, mass, density, $\delta=\delta_{s w} / \delta_{E}$ defines the relative momentum exchange thickness term [Pérezde-Tejada, 1998].

$$
\delta=\left(\frac{v_{E}}{v_{s w}}\right)^{2} \frac{n_{E} m_{E}}{n_{s w} m_{s w}}\left(1-\frac{n_{s w_{E}} v^{2}{ }_{s w_{E}}}{n_{s w} v^{2}{ }_{s w}}\right)^{-1}
$$

The typical solar wind density $n_{s w}$ and velocity $v_{s w}$ are $5 \mathrm{~cm}^{-3}$ and $400 \mathrm{~km} / \mathrm{s}$. Since solar wind particles are $\mathrm{H}^{+}$, for a dominant $\mathrm{O}^{+}$population $m_{s w} / m_{E}=1 / 16 . v_{E}$ is pick-up velocity for the terrestrial ions, using $v_{E}=10 \mathrm{~km} / \mathrm{s}$ (the Earth escape speed near the MP). From the Mars observations, the speed and density of the decelerated solar wind are $v_{s w E}=40 \mathrm{~km} / \mathrm{s}$ and $n_{s w E}=1 \mathrm{~cm}^{-3}$.

The cross section of the momentum transfer (MT) region, where the transfer of solar wind energy and momentum takes place, is defined as $A=\pi\left(R^{2}{ }_{M L B}-R^{2}{ }_{M P}\right)$, it lies between the mass loading boundary (MLB) and the magnetopause (MP) at the terminator. The MLB represents the diffuse outer boundary encompassing the region of heavy mass-loading of the solar wind by terrestrial plasma and neutral gas. The distance of MLB is $R_{M L B}=R_{M P}+\delta\left(R_{M P}-R_{E}-100 \mathrm{~km}\right)$.

Wei et al. [2014] suggested that for high values of the density of the terrestrial $\mathrm{O}^{+}$ions in the interaction region the escape rate might be significant. For example, taking $n_{E}=3 \times 10^{3} \mathrm{~cm}^{-3}$, when $R_{M P}=R_{i m b}=1.2 R_{E}$, one obtain $Q_{\text {pickup }}=1.5 \times 10^{28} \mathrm{O}^{+} / \mathrm{s}$.

\section{Conclusions}

It is shown that during the reversal of the geomagnetic field the standoff distance of the quadrupole magnetosphere is about of $3 R_{E}$ and therefore a magnetic shielding of the Earth atmosphere is still effective. The Earth's intrinsic magnetic field protects the atmosphere from sputtering and ion pickup [Lammer et al., 2007], but contributes to the polar and auroral winds through open field lines from polar caps and cusps. We assume that the main sources of polar and auroral winds are solar illumination and solar wind energy, respectively. Both escape processes are proportional to the area of open field line regions. The auroral wind also depends on the crosssectional area of interaction of the solar wind and the magnetosphere.

Since the magnetic moment of the dipole field decreases during the geomagnetic reversal, the area of the polar caps and the ion escape rate from them increases until the magnetic field configuration changes. A change in the field configuration leads to the emergence of new quadrupole equatorial belt and two polar caps, their total area 


\section{Olga 0. Tsareva et al.}

reaches a minimum when the dipole component disappears (the pure quadrupole configuration), therefore, the total ion escape rate drops sharply and then increases with decreasing quadrupole field strength (Figure 4). Table 1 gives average estimates of the escape rates of ionospheric ions at the time of reversal for the dipole and quadrupole field configurations.

The atmosphere plays a key role in protecting earthly life from radiation Tsareva et al. [2018]. Estimated the radiation doses on Earth from cosmic rays during magnetic field reversal, assuming the thickness of the Earth's atmosphere to be unchanged. According to our estimates, the escape rates of atmospheric particles during the reversal process will increase by 2.5 times (Figure 4), i. e. the atmosphere will become slightly thinner, but by the time of the reversal the escape rates will decrease by 2 times, and the atmosphere will become a little more dense. Thus, in order to estimate doses, we can assume that the Earth's atmosphere does not change on average during the reversal period.

Acknowledgements. This work was supported by Russian Science Foundation (grant \# 20-42-04418).

\section{References}

Andrè, M. and C. M. Cully (2012). Low-energy ions: A previously hidden solar system particle population, Geophys. Res. Lett., 39, L03, 101, https://doi.org/10.1029/2011g1050242.

Barabash, S. (2010). Venus, Earth, Mars: Comparative Ion Escape Rates, 12, 5308, 2010EGUGA..12.5308B

Bouhram, M., B. Klecker, W. Miyake, H. Rème, J.-A. Sauvaud, M. Malingre, L. Kistler and A. Blăgău (2004). On the altitude dependence of transversely heated $\mathrm{O}^{+}$distributions in the cusp/cleft, Ann. Geophysicae, 22, 1787-1798, https://doi.org/10.5194/angeo-22-1787-2004.

Brain, D. A., F. Leblanc, J. G. Luhmann, T. E. Moore and F. Tian (2013). Planetary magnetic fields and climate evolution. In Comparative Climatology of Terrestrial Planets, Univ. of Arizona, Tucson, https://doi.org/10.2458/azu_uapress_9780816530595-ch20.

Brain, D. A., F. Bagenal, Y.-J. Ma, H. Nilsson and G. S. Wieser (2016). Atmospheric escape from unmagnetized bodies, J. Geophys. Res.: Planets, 121, https://doi.org/10.1002/2016JE005162.

Dubinin, E., M. Fraenz, A. Fedorov, R. Lundin, N. Edberg, F. Duru, and O. Vaisberg (2011). Ion Energization and Escape on Mars and Venus, Space Sci. Rev., 162, (1), 173-211, https://doi.org/10.1007/s11214-011-9831-7.

Dubinin, E., M. Fraenz, M. Pätzold, J. McFadden, J. S. Halekas, G. A. DiBraccio, J. E. P. Connerney, F. Eparvier, D. Brain, B. M. Jakosky, O. Vaisberg and L. Zelenyi (2017), The Effect of Solar Wind Variations on the Escape of Oxygen Ions From Mars Through Different Channels: MAVEN Observations, J. Geophys. Res.: Space Physics, 122, (11), 11,285-11,301, https://doi.org/10.1002/2017JA024741.

Dungey, J. W. (1961). Interplanetary magnetic field and the auroral zones, Phys. Rev. Lett., 6, 47-48.

Engwall, E., A. I. Eriksson, C. M. Cully, M. Andrè, R. Torbert and H. Vaith (2009). Earth's ionospheric outflow dominated by hidden cold plasma, Nature Geosci., 2, (1), 24-27, https://doi.org/10.1038/ngeo387.

Fok, M.-C., Y. Ebihara and T. Moore (2005). Inner magnetospheric plasma interaction and coupling with the ionosphere, Adv. Polar Upper Atmos. Res., 19.

Glassmeier, K.-H., J. Vogt, A. Stadelmann and S. Buchert (2004). Concerning long-term geomagnetic variations and space climatology, Ann. Geophysicae, 22, (10), 3669-3677, https://doi.org/10.5194/angeo-22-36692004.

Glassmeier, K.-H., O. Richter, J. Vogt, P. Möbus and A. Schwalb (2009). The Sun, geomagnetic polarity transitions, and possible biospheric effects: review and illustrating model, Intl J. Astrobiol., 8, (3), 147159, https://doi.org/10.1017/S1473550409990073.

Glatzmaier, G. A. and P. H. Roberts (1995) A three-dimensional self-consistent computer simulation of a geomagnetic field reversal, Nature, 377, 203-209.

Gunell, H., R. Maggiolo, H. Nilsson, G. S. Wieser, R. Slapak, J. Lindkvist, M. Hamrin and J. De Keyser (2018). Why an intrinsic magnetic field does not protect a planet against atmospheric escape, Astron. Astrophys., 614, (L3), https://doi.org/10.1051/0004-6361/201832934.

Kivelson, M. G. and C. T. Russell (1995). Introduction to space physics, Cambridge University Press. 
Lammer, H., H. I. M. Lichtenegger, Y. N. Kulikov, J.-M. Grießmeier, N. Terada, N. V. Erkaev, H. K. Biernat, M. L. Khodachenko, I. Ribas and T. Penz (2007). Coronal Mass Ejection (CME) Activity of Low Mass M Stars as An Important Factor for The Habitability of Terrestrial Exoplanets. II. CME-Induced Ion Pick Up of Earth-like Exoplanets in Close-In Habitable Zones, Astrobiol., 7, (1), 185-207, https://doi.org/10.1089/ast.2006.0128.

Lammer, H., A. L. Zerkle, S. Gebauer, N. Tosi, L. Noack, M. Scherf, E. Pilat-Lohinger, M. Güdel, J. L. Grenfell, M. Godolt and A. Nikolaou (2018). Origin and evolution of the atmospheres of early Venus, Earth and Mars, Astron. Astrophys. Rev., 26, https://doi.org/10.1007/s00159-018-0108-y.

Le, G., X. Blanco-Cano, C. T. Russell, X.-W. Zhou, F. Mozer, K. J. Trattner, S. A. Fuselier and B. J. Anderson (2001). Electromagnetic ion cyclotron waves in the high-altitude cusp: Polar observations, J. Geophys. Res., 106, (A11), 19 067-19 079.

Levy, R. H., H. E. Petschek and G. L. Siscoe (1964). Aerodynamic aspects of magnetospheric flow, AIAA J., 2, 2065.

Lundin, R. and E. M. Dubinin (1992). Phobos-2 results on the ionospheric plasma escape from Mars, Adv. Space Res., 12, 255-263, https://doi.org/10.1016/0273-1177(92)90338-X.

Miyake, W., T. Mukai and N. Kaya (2000). Interplanetary magnetic field control of dayside ion conics, J. Geophys. Res., 105, (A10), 23 339-23 344, https://doi.org/10.1029/2000JA900082.

Nilsson, H., I. A. Barghouthi, R. Slapak, I. A. Eriksson and M. Andrè (2012). Hot and cold ion outflow: Spatial distribution of ion heating, J. Geophys. Res.: Space Physics, 117, 11 201, https://doi.org/10.1029/2012JA017974.

Ogawa, Y., R. Fujii, S. Buchert, S. Nozawa, S. Watanabe and A. P. Van Eyken (2000). Simultaneous EISCAT Svalbard and VHF radar observations of ion upflows at different aspect angles, Geophys. Res. Lett., 27, 81-84, https://doi.org/10.1029/1999GL010665.

Pérez-de-Tejada, H. (1998). Momentum transport in the solar wind erosion of the Mars ionosphere, J. Geophys. Res.: Planets, 103, 31499-31508, https://doi.org/10.1029/1998JE900001.

Pollock, C. J., M. O. Chandler, T. E. Moore, J. H. Waite Jr., C. R. Chappell and D. A. Gurnett (1990). A Survey of Upwelling Ion Event Characteristics, J. Geophys. Res., 95, (A11), 18 969-18 980, https://doi.org/10.1029/JA095iA11p18969.

Siscoe, G. L. and C.-K. Chen (1975). The Paleomagnetosphere, J. Geophys. Res., 80, (34), 4675-4680, https://doi.org/10.1029/ja080i034p04675.

Slapak, R., M. Hamrin, T. Pitkänen, M. Yamauchi, H. Nilsson, T. Karlsson and A. Schillings (2017). Quantification of the total ion transport in the near-Earth plasma sheet, Ann. Geophysicae, 35, (4), 869877, https://doi.org/10.5194/angeo-35-869-2017.

Stern, D. P. (1973). A Study of the Electric Field in an Open Magnetospheric Model, J. Geophys. Res., 78, (31), 7292-7305, https://doi.org/10.1029/JA078i031p07292.

Strangeway, R. J., R. E. Ergun, Y.-J. Su, C. W. Carlson and R. C. Elphic (2005). Factors controlling ionospheric outflows as observed at intermediate altitudes, J. Geophys. Res.: Space Physics, 110, A03, 221, https://doi.org/10.1029/2004ja010829.

Strangeway, R. J., C. T. Russell, J. G. Luhmann, T. E. Moore, J. C. Foster, S. V. Barabash and H. Nilsson (2010). Does a Planetary-Scale Magnetic Field Enhance or Inhibit Ionospheric Plasma Outflows? AGU Fall Meeting Abstracts, SM33B-1893.

Thebault, E., C. C. Finlay, and H. Toh (2015). International Geomagnetic Reference Field: the 12th generation, Earth, Planets and Space, 67, 158, https://doi.org/10.1186/s40623-015-0313-0.

Tian, F., E. Chassefière, F. Leblanc and D. A. Brain (2013). Atmosphere escape and climate evolution of terrestrial planets. In Comparative Climatology of Terrestrial Planets, Univ. of Arizona, Tucson, https://doi.org/10.2458/azu_uapress_9780816530595-ch23.

Tsareva, O. O. (2019) Generalization of Störmer Theory for an Axisymmetric Superposition of Dipole and Quadrupole Fields, J. Geophys. Res.: Space Physics, 124, 2844-2853, https://doi.org/10.1029/2018JA026164.

Tsareva, O. O., L. M. Zelenyi, H. V. Malova, M. V. Podlozko, E. P. Popova and V. Y. Popov (2018). What expects humankind during the inversion of the Earth's magnetic field: threats imagined and real, Phys. Usp., 61, (2), 191-202, https://doi.org/10.3367/UFNe.2017.07.038190. 


\section{Olga 0. Tsareva et al.}

Vogt, J. and B. Zieger (2004). MHD simulations of quadrupolar paleomagnetospheres, J. Geophys. Res., 109, A12, 221, https://doi.org/10.1029/2003JA010273.

Vogt, J., B. Zieger, K.-H. Glassmeier, A. Stadelmann, M.-B. Kallenrode, M. Sinnhuber and H. Winkler (2007). Energetic particles in the paleomagnetosphere: Reduced dipole configurations and quadrupolar contributions, J. Geophys. Res.: Space Physics, 112, (A6), A06 216, https://doi.org/10.1029/2006JA012224.

Voigt, G.-H. (1981). A mathematical magnetospheric field model with independent physical parameters, Planet. Space Sci. 29, (1), 1-20, https://doi.org/10.1016/0032-0633(81)90134-3.

Voigt, G.-H. (1995). Handbook of Atmospheric Electrodynamics: Volume II, CRC Press.

Wei, Y., Z. Pu, Q. Zong, W. Wan, Z. Ren, M. Fraenz, E. Dubinin, F. Tian, Q. Shi and S. Fu (2014). Oxygen escape from the Earth during geomagnetic reversals: Implications to mass extinction, Earth Planet. Sci. Lett., 394, (1-4), 94-98, https://doi.org/10.1016/j.epsl.2014.03.018.

Welling, D. T., M. Andrè, I. Dandouras, D. Delcourt, A. Fazakerley, D. Fontaine, J. Foster, R. Ilie, L. Kistler, J. H. Lee, M. W. Liemohn, J. A. Slavin, C.-P. Wang, M. Wiltberger, and A. Yau (2015). The Earth: Plasma Sources, Losses, and Transport Processes, Space Sci. Rev., 192, 145-208, https://doi.org/10.1007/s11214015-0187-2.

Yau, A. W. and M. Andrè (1997). Sources of Ion Outflow in the High Latitude Ionosphere, Space Sci. Rev., 80, (1-2), 1-25, https://doi.org/10.1023/a:1004947203046. 